\title{
Arthroscopic Treatment of Snapping Scapula Syndrome: A Case Report
}

Ahmad Faizal Roslan ${ }^{1}$, Wafiuddin Ahmad ${ }^{1}$, Faisal Amir ${ }^{2}$, Khairul Nizam Siron ${ }^{2}$

${ }^{1}$ Department of Surgical-Based, Faculty of Medicine and Health Sciences,Universiti Malaysia Sabah

2Department of Orthopedic, Traumatology and Rehabilitation, Kulliyah of Medicine, International Islamic University Malaysia.

Introduction: Snapping scapula syndrome is a condition with audible and palpable grating localized to the superomedial angle of the scapula associated with pain. The etiology is likely secondary to anomalous tissue between scapula and chest wall (e.g. bursitis, hooked superomedial angle scapula, Luschka tubercle, malunited $\mathrm{rib} / \mathrm{scapula}$ fracture and osteochondroma). Case report: We present a case of a 17year-old gentleman, with chronic pain over bilateral upper scapula associated with grating sound upon shoulders movement. Examination revealed significant audible crepitus on bilateral scapula without restriction of shoulders motion. MRI showed no abnormal finding. An attempt for conservative approach including physical therapy and steroid/local anaesthesia injection has been unsuccessful. We subsequently performed an arthroscopic bursectomy and superomedial angle scapula decompression on the right scapula. There were inflammed bursa with fibrotic tissue and prominent superomedial angle of scapula observed during the surgery. Postoperatively, the symptom over right scapula completely resolved with good patient satisfaction. At present, patient is scheduled for the similar surgery on the remaining symptomatic left scapula. In conclusion, arthroscopic scapulothoracic bursectomy with superomedial angle scapula decompression is a reliable treatment for snapping scapula syndrome with predictably high rates of pain relief, patient satisfaction as well as improvement in functional outcomes. 